\title{
Epigenetic studies revealed a Ghost proteome in PC1/3 KD macrophages under anti-tumoral resistance induced by IL10.
}

Tristan Cardon ${ }^{1}$, Bilgehan Ozcan ${ }^{1}$, Soulaimane Aboulouard ${ }^{1}$, Firas Kobeissy ${ }^{2}$, Marie Duhamel ${ }^{1}$, Franck Rodet $^{1}$, Isabelle Fournier ${ }^{1,3 *}$, Michel Salzet ${ }^{1,3 *}$

${ }^{1}$ Univ. Lille, Inserm, CHRU Lille, U-1192 - Laboratoire Protéomique, Réponse Inflammatoire et Spectrométrie de Masse-PRISM, Lille F-59000, France

${ }^{2}$ Department of Psychiatry, McKnight Brain Institute, University of Florida, Gainesville, FL 32611, USA

${ }^{3}$ Institut Universitaire de France, 75000 Paris

*Corresponding author

Michel Salzet

$\underline{\text { michel.salzet@univ-lille.fr }}$

ORCID: https://orcid.org/0000-0003-4318-0817

Isabelle Fournier:

Isabelle.fournier@univ-lille.fr

ORCID: https://orcid.org/0000-0003-1096-5044

S1 


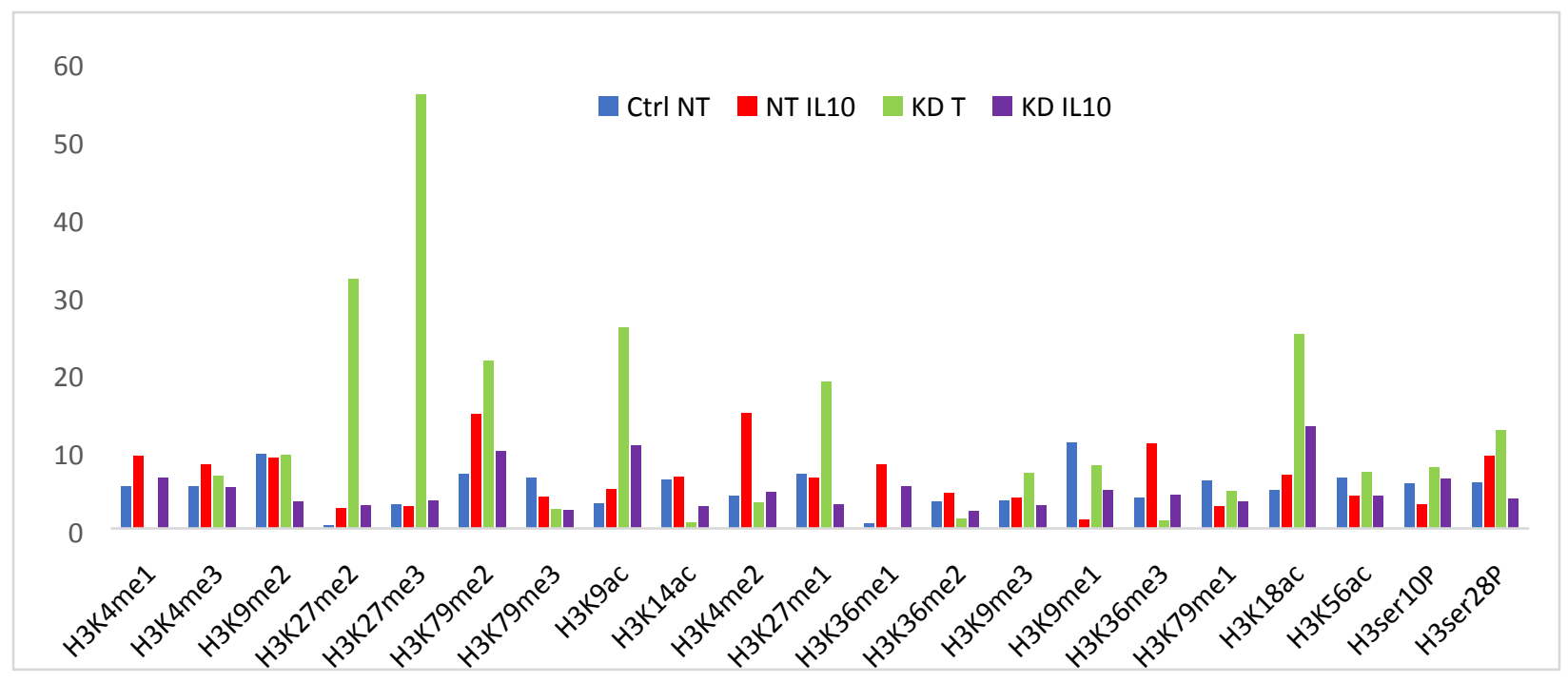

Figure S1: Quantification of post-translational changes on H3 Histones. The histones of macrophages control (NT) and PC1/3 KD stimulated with IL-10 (NT IL10 and KD IL10) -or not- were extracted. An ELISA test against post-translational changes of the H3 histones was conducted according to Epigentek's histone modification assay kit. The quantification is done by spectrophotometry at $450 \mathrm{~nm}$

S2 


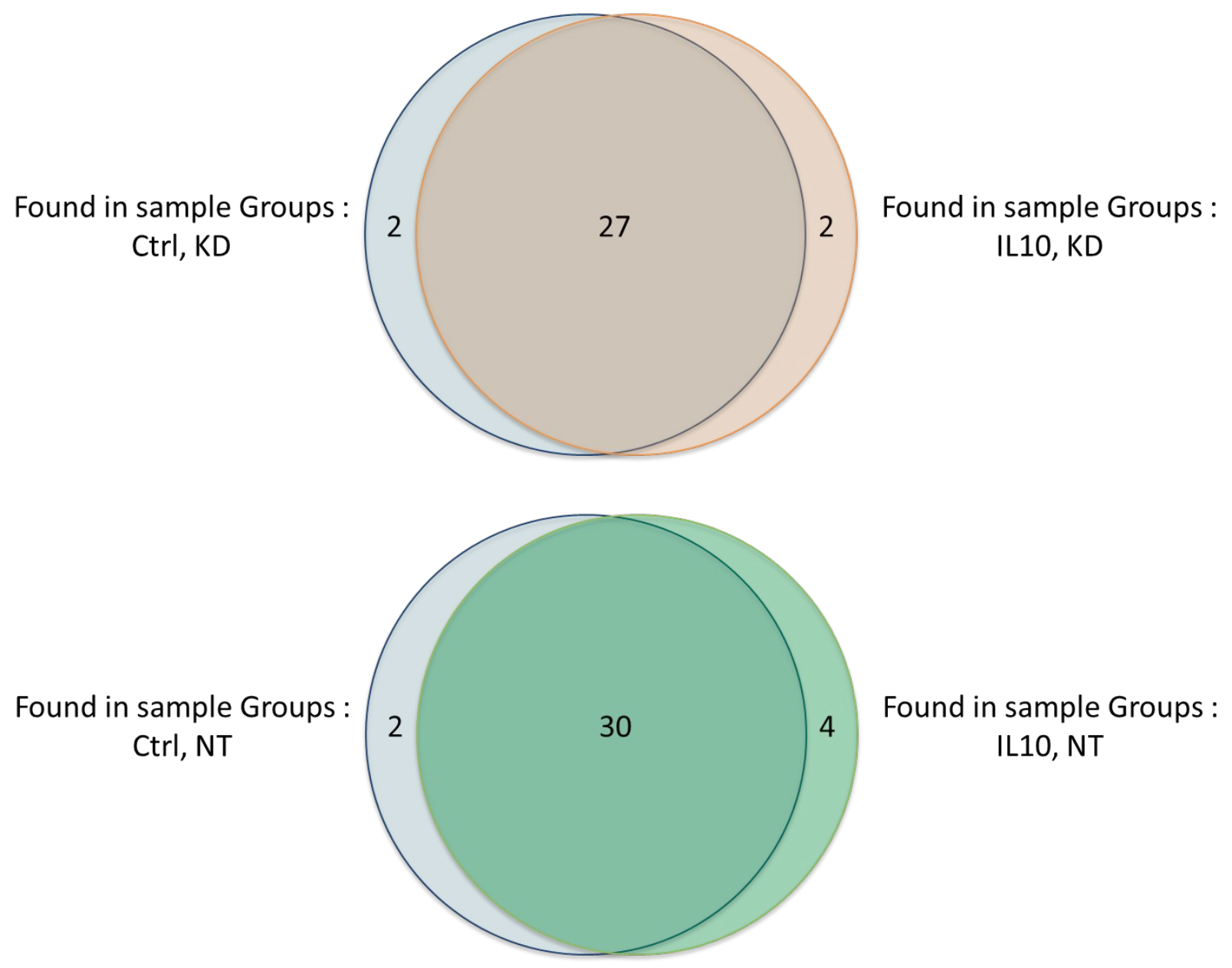

Figure S2: Venn diagram of the repartition to the identification for the different sample condition, these identifications were obtained using Proteome Discoverer 2.3

S3 